\title{
中元古代雾迷山组碳酸盐岩碳和 氧同位素组成及海平面变化
}

\section{李任伟陈锦石张淑坤 ${ }^{(4)}$}

( 中国科学院地质研究所, 北京 100029; 4中国地质科学院地质力学研究所, 北京 100083)

摘要 北京十三陵地区雾迷山组 $(1310 \sim 1207 \mathrm{Ma})$ 白云岩的 $\delta{ }^{13} \mathrm{C}$ 数值范围从- $1.5 \% \sim 1.5 \%$ (PDB), $\delta{ }^{18} 0$ 数值范围一般为- $4 \%$ - $5 \%$ (PDB). 它们碳同位素组成具有旋回性变化的特 征. $8{ }^{13} \mathrm{C}$ 从地层层序的边界发生正偏移, 至 $1.5 \%$ ( PDB), 然后发生负偏移至- $1.5 \%$ ( PDB) . 有时. 在 $\delta{ }^{13} \mathrm{C}$ 为负值的层段 $\delta{ }^{18} \mathrm{O}$ 数值较高. 约为- $\left.4.0 \% \mathrm{PDB}\right)$. 碳、氧同位素组成的这种特 征可能与海平面变化相关.

\section{关键词 碳同位素 氧同位素 中元古代 海平面变化}

在北京十三陵和河北省蓟县地区出露了两条完整的中元古界雾迷山组地层剖面. 我国地 质学家已对它们进行过大量沉积、地层和古生物的研究. 但是, 有关碳、氧同位素资料十分贫 乏. 已有的工作包括赵震 ${ }^{[1]}$ ( 1995 , 蓟县剖面, 7 个数据), 赵澄林等人 ${ }^{[2]}$ ( 1997 , 十三陵剖面, 7 个数据), Xiao Shuhai 等人 ${ }^{[3]}$ (1997, 蓟县剖面, 13 个数据). 本文作者对十三陵剖面进行了比较 系统的碳和氧同位素测定, 发现它保留了碳酸盐岩沉积时原始同位素组成的信息. 据此, 可以 追踪当时海平面的变化, 了解古海洋的碳、氧同位素组成特征. 由于地质年代古老和缺乏理想 地层剖面等原因, 在全世界范围内都缺乏中元古界沉积地层碳、氧同位素的系统资料, 因而本 文的研究成果有助于了解该时期全球海洋碳、氧同位素组成和演化特征, 发展以稳定同位素为 主要对比手段的化学地层学 ${ }^{[4]}$.

\section{1 地质背景}

十三陵地区雾迷山组地层剖面位于北京城西北约 $35 \mathrm{~km}$. 根据赵澄林等人 ${ }^{[2]}$ 对该剖面进 行的测量, 它厚达 $2168 \mathrm{~m}$, 几乎全部为白云岩. 按照岩性和韵律特征, 他们把该剖面雾迷山组 划分成 4 段. 按自下而上的顺序, 第一段由泥云岩、纹层藻叠层白云岩、藻团和锥状叠层白云 岩、层纹状燧石纹层白云岩组成; 第二段为含燧石隐晶白云岩, 纹层状白云岩、藻团和锥状白云 岩; 第三段主要由深灰色密纹层藻叠层白云岩, 凝块状藻团白云岩组成; 第四段为灰色、含燧石 条带的隐晶和微晶白云岩. 他们认为, 这 4 个段分别代表 4 个三级层序, 除第一段与下覆地层 杨庄组和第四段与上覆地层洪水庄组的界面为 iv 类层序界面外, 其他界面都为 是类层序界面; 每个层序由海侵体系域(TST) 和高位体系域(HST) 所组成, 海侵体系域包括潮下环境沉积体 系, 高位体系域包括潮坪环境沉积体系 ${ }^{[2]}$.

于荣炳 ${ }^{[5]}$ 曾给出过雾迷山组地层的年龄数据, 它与下覆杨庄组界限年龄值为 $1400 \mathrm{Ma}$, 与 上覆洪水庄组界限年龄值为 $1220 \mathrm{Ma}$. 后者的根据为洪水庄组底部伊利石的 $\mathrm{K}-\mathrm{Ar}$ 年龄测定 (1 $221 \mathrm{Ma}$ ). 王松山等人 ${ }^{[6]}$ 采用 ${ }^{40} \mathrm{Ar} /{ }^{39} \mathrm{Ar}$ 阶段加热技术和 $\mathrm{Cr}-\mathrm{Ar}$ 相关性较正方法消除次生流 体包体及过剩 $\mathrm{Ar}$ 的干扰, 获得了近杨庄组底界和距杨庄组-雾迷山组界面约 $20 \mathrm{~m}$ 处条带状燧 石岩的 ${ }^{40} \mathrm{Ar} /{ }^{39} \mathrm{Ar}$ 等时年龄值, 从此, 推测雾迷山组下界年龄值应为 $(1310 \pm 20) \mathrm{Ma}$; 他们还直接 
测得了雾迷山组顶部、紧靠它与洪水庄组界面的条带状燧石的 ${ }^{40} \mathrm{Ar} /{ }^{39} \mathrm{Ar}$ 等时线年龄值为 (1 $207 \pm 10) \mathrm{Ma}^{[6]}$. 王松山等人 ${ }^{[6]}$ 所取条带状燧石岩应为沉积或早期成岩成因, 所测年龄值 可能更具有真实性.

\section{2 取样和分析方法}

本文研究工作的野外考察和取样主要参考由赵澄林等人所建立的地层剖面. 在雾迷山组 共采样品 88 个, 雾迷山组第一段 18 个, 第二段 19 个, 第三段 19 个, 第四段 32 个.

本文中碳酸盐岩为白云岩, 其碳、氧稳定同位素分析方法如下: 岩石被磨至 200 目之下, 采 用无水正磷酸溶样, 在温度 $25^{\circ} \mathrm{C}$ 时, 恒温 $72 \mathrm{~h}$, 然后将所获得的 $\mathrm{CO}_{2}$ 进行质谱分析, 分析结果采 用 PDB 标准, 以 $\delta$ 值表示.

为了判断雾迷山组碳酸盐岩碳、氧同位素组成是否遭受过沉积期后的变化, 还需要化学元 素分析的资料. 为此, 在各段选择代表性样品, 用 $\mathrm{HF} / \mathrm{HNO}_{3}$ 溶样, 采用 ICP 光谱方法进行分 析.

\section{3 结果和讨论}

\section{1 原始性检验}

在表 1 中列入了雾迷山组碳、氧同位素分析结果. 图 1 展示碳、氧同位素组成沿地层剖面 的变化. 在讨论它们的地质意义之前, 必须首先对其原始性进行检验, 即考查它们是否遭受过 沉积期后的变化. 对于如前寒武纪这样古老沉积地层的同位素地质研究, 分析数据的原始性 检验是一项基础性工作, 否则将会导致混乱甚至错误的结论. 本文将采取两种常用的标准, 即 根据微量元素的含量和碳、氧同位素组成的变化来进行检验. 由于沉积期后、特别是大气水循 环的影响, 碳酸盐岩将发生 $\mathrm{Sr}$ 的损失和 $\mathrm{Mn}$ 的加入 ${ }^{[7]}$, 因此, 可利用 $\mathrm{Mn} / \mathrm{Sr}$ 比值来判断碳同位 素组成是否遭受变化. Kaufman 等人 ${ }^{[4]}$ 提出 $\mathrm{Mn} / \mathrm{Sr}<10$ 的碳酸盐岩(包括灰岩和白云岩) 通常 可以保留其原始的碳同位素组成. 更严格的标准是 $\mathrm{Mn} / \mathrm{Sr}<2 \sim 3$, 它甚至被用于判断碳酸盐 岩的 $\mathrm{Sr}$ 同位素组成是否可代表原始的记录 ${ }^{[8,9]}$. 表 2 列入了雾迷山组若干样品的化学分析结 果, 人们从中可以发现所有白云岩样品的 $\mathrm{Mn} / \mathrm{Sr}$ 的比值都小于 10 , 多数的 $\mathrm{Mn} / \mathrm{Sr}$ 比值小于或 接近 2 3. 表 1 中 D140 和 D113(燧石岩) 具有高的 Mn/ Sr 比值, 其碳同位素组成可能遭受了 沉积期后的变化. 岩石中碳酸盐的氧同位素组成对沉积期后的变化最为灵敏, 它们若与大气 和热水流体发生同位素交换, 其 $\delta{ }^{18} \mathrm{O}$ 数值将明显减低. 按照 Kaufman 等人 ${ }^{[4]}$ 提出, 并为 Xiao 等人 (1997) 应用的判别标准, 当 $\delta^{18} \mathrm{O}$ 小于- 5 时的岩石已遭受沉积期后变化, 当 $\delta{ }^{18} \mathrm{O}$ 小于 -10 时的数据已不能使用. 若 $\delta^{18} \mathrm{O}$ 和 $\delta^{13} \mathrm{C}$ 的数值同步降低, 例如表 1 中从 D106 到 D125 的 地层段 (大致相当于赵澄林等人所描述的雾迷山组第二段), 只有那些最富 ${ }^{13} \mathrm{C}$ 和 ${ }^{18} \mathrm{O}$ 的岩石, 例 如 D109, D111, D112, D114 和 D116 (表 1)，其碳和氧同位素组成的资料较为可靠 ${ }^{[4]}$. 对于如 D124, D119, D117 和 D113 那类样品(表 1), 不仅其氧同位素组成, 而且碳同位素组成也可能遭 受了沉积期后的改造. D149 也可能遭受过类似的沉积期后的改造.

\section{2 碳、氧同位素组成特征与海平面变化}

根据 3.1 的讨论, 样品 D149, D124, D119, D117 和 D113 的碳同位素组成遭受过沉积期后的 变化, D97 的 $\delta{ }^{13} \mathrm{C}$ 值负达- 3.0\%, 虽然其 $\mathrm{Mn} / \mathrm{Sr}<3$, 也可能含有早期成岩作用过程中来自有 机碳的成分 ${ }^{[10]}$. 排除这些样品后, 我们发现雾迷山组白云岩的 $\delta^{13} \mathrm{C}$ 值落在 $-1.5 \%$ 
表 1 雾迷山组白云岩碳、氧同位素分析资料

\begin{tabular}{|c|c|c|c|c|c|c|c|}
\hline 地层 & 样品编号 & $8{ }^{13} \mathrm{C} / \%$ & $\delta{ }^{18} \mathrm{O} / \%$ & 地层 & 样品编号 & $8{ }^{13} \mathrm{C} / \%$ & $\delta^{18} \mathrm{O} / \%$ \\
\hline & DW8 & 0.93 & -5.72 & \multirow{6}{*}{$\begin{array}{l}\text { 雾迷山 } \\
\text { 组三段 }\end{array}$} & D137 & 0.21 & -4.18 \\
\hline & DW7 & 1.23 & -5.58 & & D135 & 0.27 & -5.56 \\
\hline & DW6 & 1.61 & -4.39 & & D133 & 0.60 & -5.91 \\
\hline & DW5 & 1.32 & -3.69 & & D132 & 0.32 & -5.28 \\
\hline & DW4 & 0.71 & -7.10 & & D131 & 0.88 & -6.59 \\
\hline & DW3 & 1.35 & -5.56 & & D130 & 0.01 & -5.19 \\
\hline \multirow{26}{*}{$\begin{array}{l}\text { 雾迷山 } \\
\text { 组四段 }\end{array}$} & DW2 & 0.23 & -3.22 & \multirow{24}{*}{$\begin{array}{l}\text { 雾迷山 } \\
\text { 组二段 }\end{array}$} & D125 & 0.13 & -5.37 \\
\hline & DW1 & 0.32 & -3.16 & & D124 & -3.73 & -12.19 \\
\hline & DW 181 & 0.64 & -3.24 & & D123 & -0.25 & -6.68 \\
\hline & DW 180 & 0.18 & -4.10 & & D122 & 0.23 & -5.87 \\
\hline & DW 179 & -0.19 & -4.23 & & D121 & -0.70 & -5.65 \\
\hline & DW 178 & 0.28 & -4.42 & & D120 & -0.44 & -4.74 \\
\hline & DW 177 & 0.40 & -4.36 & & D119 & -1.44 & -4.77 \\
\hline & DW 176 & 0.75 & -5.22 & & D118 & -0.04 & -3.80 \\
\hline & DW 175 & 0.00 & -4.20 & & D117 & -1.34 & -8.15 \\
\hline & DW 174 & 0.29 & -4.44 & & D116 & 0.24 & -4.49 \\
\hline & DW 173 & 0.08 & -3.43 & & D115 & 0.05 & -5.79 \\
\hline & DW 172 & 0.03 & -2.55 & & D114 & 0.46 & -3.57 \\
\hline & DW 171 & 0.01 & -6.46 & & D113 & -1.07 & -7.37 \\
\hline & DW169 & 0.04 & -3.62 & & D98 & -0.58 & -6.11 \\
\hline & DW168 & -0.02 & -3.52 & & D97 & -3.03 & -5.08 \\
\hline & DW 166 & -0.64 & -5.14 & & D95 & 0.31 & -4.99 \\
\hline & DW 165 & -0.38 & -4.74 & & D93 & 0.23 & -5.19 \\
\hline & DW 164 & -0.54 & -5.16 & & D92 & 0.85 & -4.68 \\
\hline & DW 163 & 0.25 & -4.31 & & D89 & 0.87 & -4.61 \\
\hline & DW 162 & -0.36 & -4.19 & & D88 & 1.01 & -4.42 \\
\hline & DW161 & 0.13 & -5.04 & & D83 & 0.91 & -4.30 \\
\hline & DW 160 & 0.05 & -4.78 & & D82 & 1.13 & -3.80 \\
\hline & D158 & 0.32 & -4.62 & & D81 & 0.54 & -5.45 \\
\hline & D157 & 0.29 & -4.07 & & D80 & 1. 44 & -4.30 \\
\hline & D156 & -0.21 & -3.72 & & D112 & -0.01 & -4.02 \\
\hline & D155 & -0.69 & -5.28 & & D111 & 0.36 & -4.30 \\
\hline \multirow{13}{*}{$\begin{array}{l}\text { 雾迷山 } \\
\text { 组三段 }\end{array}$} & D154 & -0.93 & -3.51 & \multirow{11}{*}{$\begin{array}{l}\text { 雾迷山 } \\
\text { 组一段 }\end{array}$} & D109_b & -0.01 & -4.96 \\
\hline & D153 & -1.08 & -4.55 & & D109_a & -0.09 & -3.76 \\
\hline & D152 & -0.84 & -4.17 & & D108 & -0.16 & -5.38 \\
\hline & D151 & -0.43 & -4.68 & & D106 & 0.26 & -4.09 \\
\hline & D149 & -1.50 & -5.69 & & D105 & -1.44 & -4.78 \\
\hline & D147 & -0.95 & -4.20 & & D104 & -0.90 & -4.81 \\
\hline & D146 & -0.94 & -4.13 & & D103 & -1.14 & -5.09 \\
\hline & D145 & -0.88 & -4.72 & & D102 & -0.74 & -4.35 \\
\hline & D144 & -1.35 & -4.03 & & D101 & -0.94 & -3.96 \\
\hline & D143 & -1.00 & -3.65 & & D100 & -0.89 & -6.56 \\
\hline & D141 & -0.21 & -3.88 & & D99 & -0.67 & -4.94 \\
\hline & D140 & -1.00 & -3.65 & \multirow[t]{2}{*}{ 杨庄组 } & & $-2.33^{\mathrm{a})}$ & $-3.56^{\mathrm{a})}$ \\
\hline & D139 & 1.38 & -4.66 & & & & \\
\hline
\end{tabular}

a) 杨庄组顶部数据取自文献[2] 
1. $5 \%$ 的范围, 而且具有旋回性变化的特征 (图 1). 在雾迷山组第一段, $\delta^{13} \mathrm{C}$ 值从杨庄组与雾 迷山组界限附近的负值 $(-2.3 \%$ 见文献 [2] ) 向正 值偏移至 $1.4 \%$, 然后通过接近零值向负值偏移, 并降至- $1.4 \%$ ，雾迷山组第三段具有相似的变 化. $\delta{ }^{13} \mathrm{C}$ 在旋回的前半部为正值, 在后半部为负 值. 按赵澄林等人 ${ }^{[2]}$ 研究, 这两段地层各自代表 一个三级层序, 并都由海侵和高位体系域组成. 由于这些三级层序的时限明显超过 Vail 等人 ${ }^{[11]}$ 所限定的范围 0.5 $3 \mathrm{Ma}$, 人们对其划分可能存在 疑义. 但是, 他们关于这两段地层海平面变化的 推论与本文所揭示的碳同位素组成特征相吻合: 即大致相当于海侵体系域沉积的碳酸盐岩(表 1 中雾迷山组第一段 D80 D95 和雾迷山组第三段 D130 D139) 具有正的 $\delta{ }^{13} \mathrm{C}$ 数值, 相当于高位体 系域沉积的碳酸岩 (表 1 中雾迷山组第一段 D97 D106) 具有负的 $\delta{ }^{13} \mathrm{C}$ 值. 表 1 中雾迷山组 第三段 D140 D154 具有负的 $\delta{ }^{13} \mathrm{C}$ 值, 它们也可 能沉积於高位体系域.

十三陵地区雾迷山组碳酸盐岩氧同位素组成 的变化远较碳同位素复杂. 但是, 若删除遭受过 沉积期后变化的样品, 它们仍呈现一定的规律. 其一是绝大多数样品的 $\delta^{18} \mathrm{O}$ 值都落在一个狭窄 的范围: - 4\%o - 5\%o(图 1). Veizer 等人 ${ }^{[12,13]}$ 曾研究过古元古代碳酸盐岩的氧同位素组成, 他 们认为白云岩的最佳 $\delta^{18} \mathrm{O}$ 数值为- $4 \%$ - $5 \%$, 低于显生宙. 雾迷山时期( 1 310 $1207 \mathrm{Ma}$ ) 白云 岩的氧同位素组成与古元古代一致, 这意味着它 们古海洋的氧同位素组成也可能具有相似性. 此

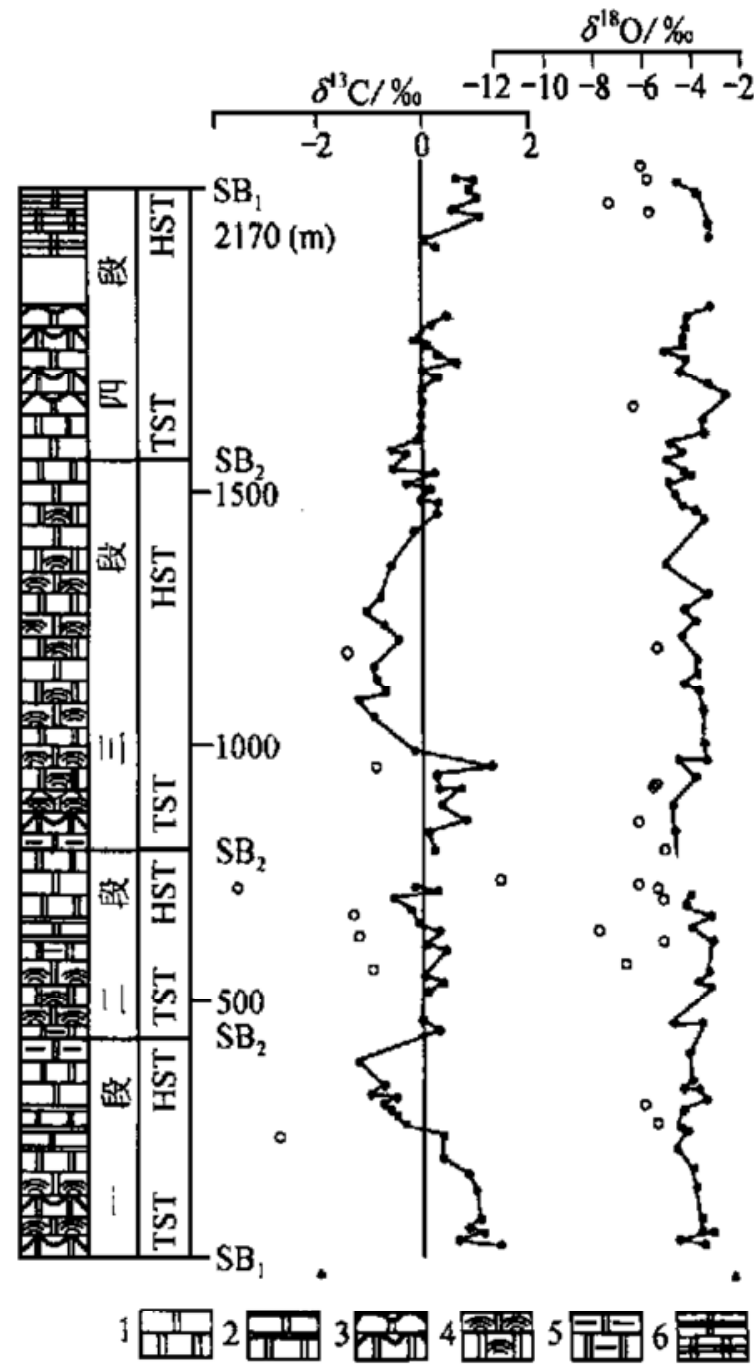

图 1 北京十三陵地区雾迷山组成碳、氧 同位素组成及演化

1 一隐晶白云岩, 2 一含细条带燧石白云岩, 3 一含 不规则条带燧石白云岩, 4 一一藻叠层石白云岩, 5 泥白云岩, 6 一纹层状白云岩. 空心圆示同位素组成 遭受了沉积期后的变化, 三角形数据取自文献[2]. $\mathrm{SB}_{1}$ 示 iv型层序界面, $\mathrm{SB}_{2}$ 示 $€$ 型层序界面, TST 示海侵体系 域,HST 示高位体系域(层序和分段根据文献[2]) 外, 雾迷山组第三段形成於海侵体系域的岩石 (表 1 中 D130 D139) 具有低的 $\delta{ }^{18} \mathrm{O}$ 数值, 多数 大于或近似 $-5.0 \%$, 相反, 可能形成於高位体系域的岩石 (表 1 中 D140 D154) 其 $\delta^{18} \mathrm{O}$ 数值 较高, 多数从- $3.5 \%$ - $4.7 \%$, 平均为 $-4.0 \%$ 。

\section{3 生物地球化学含义}

中元古代雾迷山时期碳酸盐岩相对具有重碳同位素组成的层段与海侵相关. 可以在新元 古代 ${ }^{[4]}$, 也可以在显生宙找到类似的例子. 在白严纪森诺曼阶-土仓阶 ${ }^{[14 、 15]}$ 、早侏罗世 ${ }^{[16]}$ 、中 奥陶世晚期 ${ }^{[17]}$ 以及晚寒武世 ${ }^{[18,19]}$, 人们都可以在海侵时期沉积的碳酸盐岩地层中发现 $\delta^{13} \mathrm{C}$ 数值的正偏移. 原因在于此时的海洋有较高的有机产率和高的有机碳埋藏速率, 由于沉积有 
表 2 雾迷山组白云岩部分元素化学分析资料

\begin{tabular}{crrrr}
\hline 样品编号 & $\mathrm{CaO} / \%$ & $\mathrm{MgO} / \%$ & $\mathrm{Al}_{2} \mathrm{O}_{3} / \%$ & $\mathrm{Mn} / \mathrm{Sr}$ \\
\hline $\mathrm{DW} 5$ & 28.93 & 20.78 & 0.21 & 4.9 \\
$\mathrm{DW} 4$ & 29.31 & 20.05 & 0.39 & 3.6 \\
$\mathrm{DW} 168$ & 28.80 & 20.20 & 0.04 & 2.9 \\
$\mathrm{DW} 157$ & 28.90 & 20.50 & 0.00 & 1.8 \\
$\mathrm{D} 155$ & 28.10 & 19.80 & 0.02 & 3.2 \\
$\mathrm{D} 140^{\mathrm{a})}$ & 0.89 & 0.60 & 0.14 & 75.6 \\
$\mathrm{D} 139$ & 26.50 & 18.74 & 0.01 & 2.1 \\
D131 & 28.90 & 20.30 & 0.01 & 2.5 \\
D124 & 20.00 & 9.10 & 0.20 & 1.5 \\
$\mathrm{D} 114$ & 27.20 & 19.23 & 0.01 & 2.5 \\
D113 & 7.90 & 5.32 & 0.02 & 19.9 \\
D102 & 29.00 & 20.80 & 0.01 & 1.8 \\
D97 & 29.10 & 21.10 & 0.00 & 2.5 \\
D93 & 28.80 & 20.10 & 0.00 & 3.3 \\
D92 & 28.70 & 20.50 & 0.04 & 3.8 \\
D88 & 27.60 & 19.86 & 0.03 & 6.8 \\
D80 & 26.90 & 19.30 & 0.00 & 3.5 \\
\hline
\end{tabular}

a) D140 为燧石岩, D113 为含碳酸盐的燧石岩

机质从海洋中选择地吸收了轻碳同位素 $\left({ }^{12} \mathrm{C}\right)$, 结果造成海洋碳酸盐库对重碳同位素 $\left({ }^{13} \mathrm{C}\right)$ 的相 对富集. 同样可以用这种机制来解释雾迷山组地层中所观察到的碳同位素演化特征, 区别仅 在于上述显生宙时期, 海洋碳库的变化要剧烈得多, 甚至引起了全球环境和气候的变化. 大量 有机质的埋藏将导致大气圈中 $\mathrm{O}_{2}$ 含量的增加和 $\mathrm{CO}_{2}$ 含量的减少, 并最终导致全球气候变冷 (冰室效应)、甚至某些生物的绝灭. Patterson 等人 ${ }^{[19]}$ 发现在现代碳酸盐台地, 由于有机质呼吸 作用释放出轻同位素的 $\mathrm{CO}_{2}$, 结果会造成滞流在台地上的海水较之广海水贫 ${ }^{13} \mathrm{C}$. 此外, 在宾夕 法尼亚 ( 中-上石炭统) 向上变浅的沉积体系中保存完好的腕足类不仅贫 ${ }^{13} \mathrm{C}$, 而且富 ${ }^{18} \mathrm{O}$, Patterson 等人 ${ }^{[19]}$ 认为其原因在于碳酸盐台地上海水的滞流和蒸发作用. 也可以根据上述机构来解 释雾迷山组的碳、氧同位素组成特征. 若形成於“高位体系域”时期的碳酸盐岩处於潮坪环境, 由于微生物对有机质的呼吸作用向滞流的海水释放富轻碳同位素的 $\mathrm{CO}_{2}$, 而蒸发作用又使其 富 ${ }^{18} \mathrm{O}$, 最终就在碳酸盐岩石中产生了贫 ${ }^{13} \mathrm{C}$ 和富 ${ }^{18} \mathrm{O}$ 的同位素记录.

本文通过同位素和微量元素地球化学以及沉积学的研究, 首次证明在北京十三陵地区中 元古代雾迷山组碳酸盐岩中保留有原始沉积的碳、氧同位素记录. 白云岩的 $\delta{ }^{13} \mathrm{C}$ 数值范围 从- $1.5 \%$ + $1.5 \%, \delta{ }^{18} \mathrm{O}$ 数值范围大多为- 4\%o - $5 \%$ a 碳同位素组成具有旋回性变化的 特征, ${ }^{13} \mathrm{C}$ 从层序边界的负值发生正偏移升至约 $1.5 \%$, 然后发生负偏移, 降至约- $1.5 \%$ a 有 时, 在 $\delta{ }^{13} \mathrm{C}$ 为正值的层段, $\delta{ }^{18} \mathrm{O}$ 可为相对低的数值 (约为- $5.0 \%$ ), 在 $\delta{ }^{13} \mathrm{C}$ 为负值的层段, $\delta^{18} \mathrm{O}$ 可为较高值 (约为- $4.0 \%$ ). 雾迷山组这种碳、氧同位素组成特征可以反映海平面的变 化. 在海侵体系域时期海水有相对高的有机产率和有机碳埋藏速率, 在高位体系域时期的碳 酸盐台地可能为滞流和蒸发环境, 海水中微生物因呼吸而消耗有机质的作用比较强烈.

致谢陈志明曾参与本文的野外工作,闻传芬帮助完成碳、氧同位素分析, 唧桂枝帮助完成化学分析, 作者表 示感谢. 本工作为国家自然科学基金 (批准号: 49573199) 资助项目. 


\section{参 考 文 献}

1 赵 震. 从氧碳同位素组成看蓟县元古宙碳酸盐岩特征. 沉积学报, 1995, 13(3) : 46 53

2 赵澄林, 李儒峰, 周劲松. 华北中新元古界油气地质与沉积学. 北京: 地质出版社, 1997

3 Xiao Shuhai, Knoll A H, Kaufman A J, et al. Neoproterozoic fossils in Mesoproterozoic rocks? Chemostratigraphic resolution of a biostratigraphic conundrum from the North China Platfrom. Precambrian Res, 1997, 84: 197 220

4 Kaufman A J, Knoll A H. Neoproterozoic variations in the C- isotope composition of seawater: stratigraphic and biogeochemical implications. Precambrian Res, 1995, 73: 27 49

5 于荣柄, 张学祺. 燕山地区晚前寒武纪同位素地质年代学研究. 见: 天津地质矿产研究所所刊(11). 北京: 地质出版社, 1984. $1 \sim 23$

6 王松山, 桑海清, 䙑 翼, 等. 蓟县剖面扬庄组和雾迷山组形成年代的研究成果. 地质科学, 1995, 30(2): 166 172

7 Veizer J. Chemical diagenesis of carbonates: theory and application. In: Arthur M A, Aanderson T F, Kaplan I R, et al, eds. Stable Isotopes in Sedimentary Geology. S E P M Sthort Course, 1983, 10: 3 100

8 Derry L A, Kaufman A J, Jacobsen S B. Sedimentary cycling and environmental change in the Late Proterozoic: evidence trom stable and radiogenic isotopes. Geochim Cosmochim Acta, 1992, 56: 1317 1329

9 Kaufman A J, Jacobsen S B, Knoll A H. The Vendian record of Sr-and Cisotopic variations in searwater: implications for tectonics and paleoclimate. Earth Planet Sci Lett, 1993, 120: 409 430

10 Irwin H, Curtis C D, Coleman M L. Isotopic evidence for source of diagenetic carbonates formed during burial of organic rich sediments. Nature, 1977, 269: 209 313

11 Vail P R, Audemard F, Bowman S A, et al. The stratigraphic signatures of tectonics, eustacy and sedimentology- an overview. Einsele G, Ricken W, Seilacher A, eds. Cycles and Events in Stratigraphy. Berlin: Springer-Verlag, 1991. 617 659

12 Veizer J, Clayton R N, Hinton R W. Geochemistry of Precambrian carbonates. IV. early paleoproteroic(2. $25 \pm 0.25$ Ca) seawater. Geochim Cosmochim Acta, 1992, 56: 875 885

13 Veizer J, Plumb K A, Clayton R N. Geochemistry of Precambrian carbonates. (九) late paleoproterozoic seawater. Geochim Cosmochim Acta, 1992, 56: 2487 2501

14 Scholle P A, Arthur M A. Carbon isotope fluctuations in Cretaceous pelagic limestones: potential stratigraphic and petroleum exploration tool. A A P G Bull, 1980, 64: 67 87

15 Arthur M A, Dean W E, Pratt L M. Geochemical and climatic effects of increased marine organic carbon burial at the Cenomanian/Turonian boundary. Nature, 1988, 335: 714 717

16 Jenkyns H C, Clayton C J. Lower Jurassic epicontinental carbonates and mudstones from England, Wales: chemostratigraphic signals and the early Toarcian anoxic event. Sedimentology, 1977, 44: 687 706

17 Patzkowsky M E, Slupik L M, Arthur M A, et al. Late Middle Ordovician environmental change and extinction: Harbinger of the Late Ordovician or continuation of Cambrian patterns? Geology, 1997, 25: 911 914

18 Saltzman M R, Runnegar B, Lohmann K C. Carbon isotope stratigraphy of Upper Cambrian(Steptoean Stage) sequences of the eastern Great Basin: record of a global oceanographic event. GSA Bulletin, 1988, 110: 285 297

19 Patterson W P, Walter L M. Depletion of ${ }^{13} \mathrm{C}$ in seawater $\mathrm{CO}_{2}$ on modem carbonate platforms: significance for the carbon isotopic record of carbonates. Geology, 1994, 22: 885 888 